\title{
Review of: "A face recognition software framework based on principal component analysis"
}

Wudneh Simegn

Potential competing interests: The author(s) declared that no potential competing interests exist.

This paper is highly important for readers 